Supporting Information for

\title{
Is Ice Nucleation by Organic Crystals Non-Classical? An Assessment of the Monolayer Hypothesis of Ice Nucleation.
}

\author{
Atanu K. Metya ${ }^{1}$ and Valeria Molinero ${ }^{1 *}$ \\ ${ }^{1}$ Department of Chemistry, The University of Utah, Salt Lake City, Utah 84112-0850, USA \\ *corresponding author, email: Valeria.Molinero@utah.edu
}

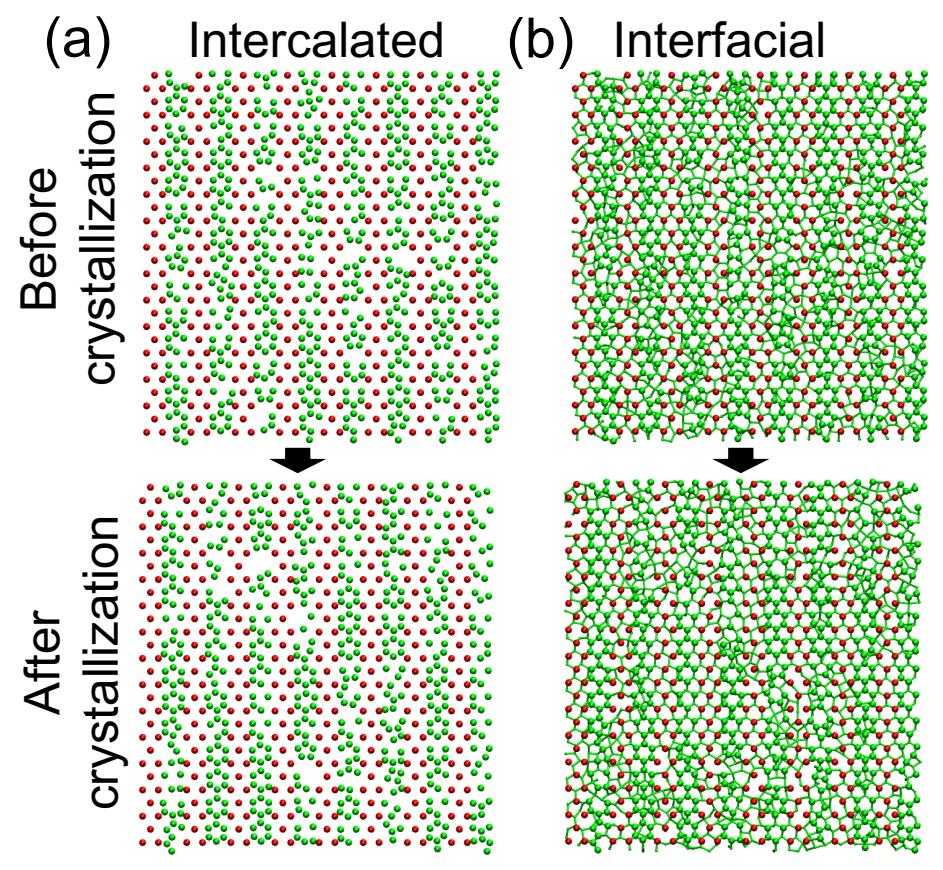

Fig. S1. Ordering of water on the (100) face phloroglucinol dihydrate crystal, which is only marginally able to nucleate ice. Snapshots before and after ice crystallization of (a) intercalated (b) interfacial water from a cooling simulation. We here define the intercalated water as those within $0.2 \mathrm{~nm}$ for the plane of $\mathrm{OH}$ groups and interfacial water as those within $0.5 \mathrm{~nm}$ from the $\mathrm{OH}$ plane of this face. The cutoffs distances are slightly larger than we consider for the other faces because the aromatic groups exposed in this surface prevent water from getting very close to the plane of the $\mathrm{OH}$. 

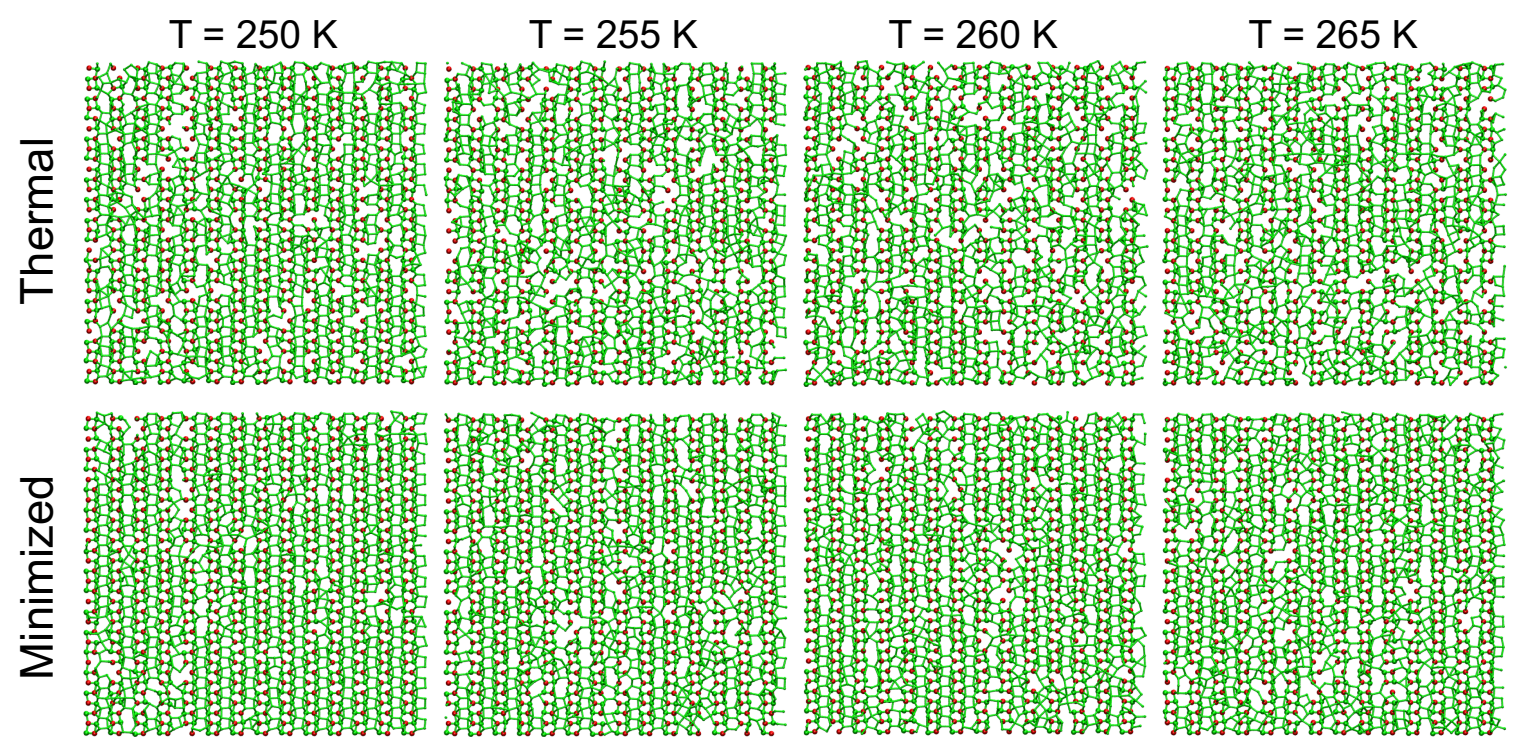

Fig. S2. Ordering of interfacial water on the (010) faces of the phloroglucinol dihydrate crystal as a function of temperature, starting from a configuration equilibrated at $250 \mathrm{~K}$. The snapshots correspond to the last configuration after $20 \mathrm{~ns}$ of run at the corresponding temperature. We do not find any significant difference in the ordering if the initial configuration at a given temperature if the initial structure was equilibrated at 250 or $300 \mathrm{~K}$. Hydroxyl groups of crystal and water molecules are denoted by red and green balls, respectively. We define the interfacial water as those within $0.35 \mathrm{~nm}$ for the plane of $\mathrm{OH}$ groups.

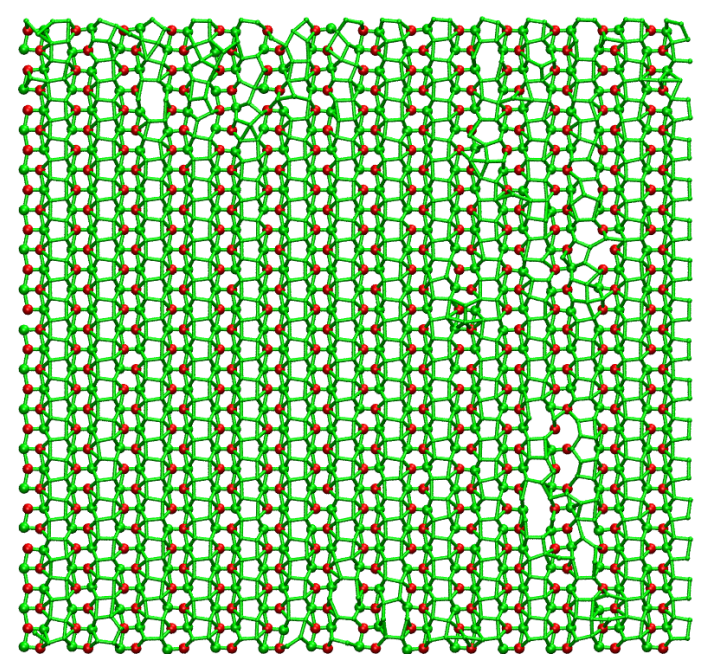

Fig. S3. Structure of the (010) face of phloroglucinol dihydrate covered with the rigid monolayer used for the icebiasing simulations. Note that as the monolayer was obtained from a simulation in which the crystallization was not complete (to avoid the structural transformation of interfacial water) there are defects that probably prevent the complete spreading of ice on it. Hydroxyl groups of crystal and water molecules are denoted by red and green balls, respectively. We define the interfacial water as those within $0.35 \mathrm{~nm}$ for the plane of $\mathrm{OH}$ groups. 
(a) Initial config. $\mathrm{T}=260 \mathrm{~K}$

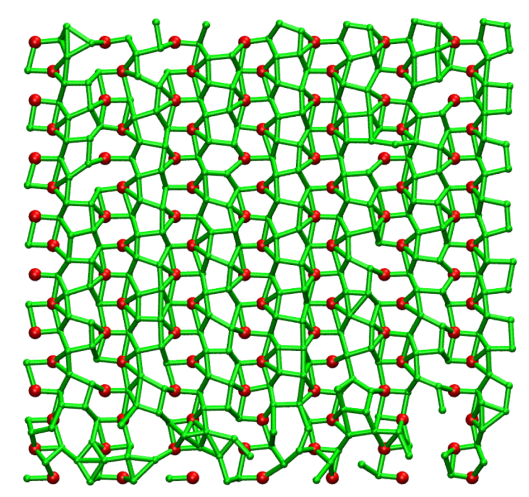

(b) Final config. $\mathrm{T}=265 \mathrm{~K}$

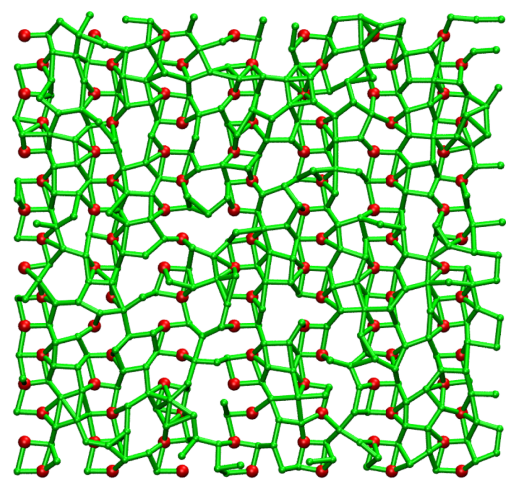

(c) Final config. $\mathrm{T}=270 \mathrm{~K}$

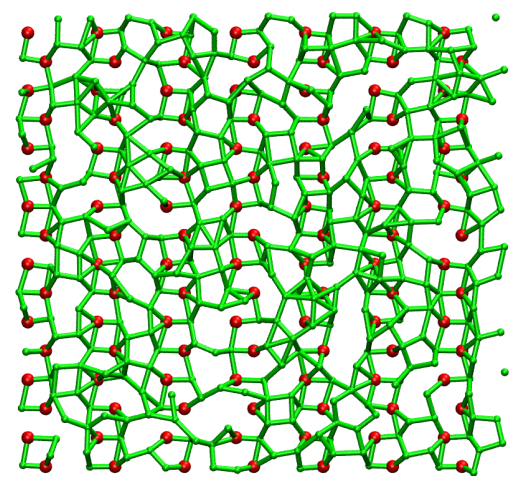

Fig. S4. Ordering of interfacial water on the (010) face of phloroglucinol dihydrate surface modeled with the allatom model of phloroglucinol and TIP4P/Ice water model. (a) The starting structure of interfacial water at 260K, taken from an ice crystal where ice has been crystallized with a bias in the global bond order parameter. (b) and (c) the snapshots of ordering of interfacial water at the end of $10 \mathrm{~ns}$ simulations at the mentioned temperature, in these cases starting simulation from configuration (a). The green sticks connect water and $\mathrm{OH}$ groups (red balls) within $0.35 \mathrm{~nm}$. 


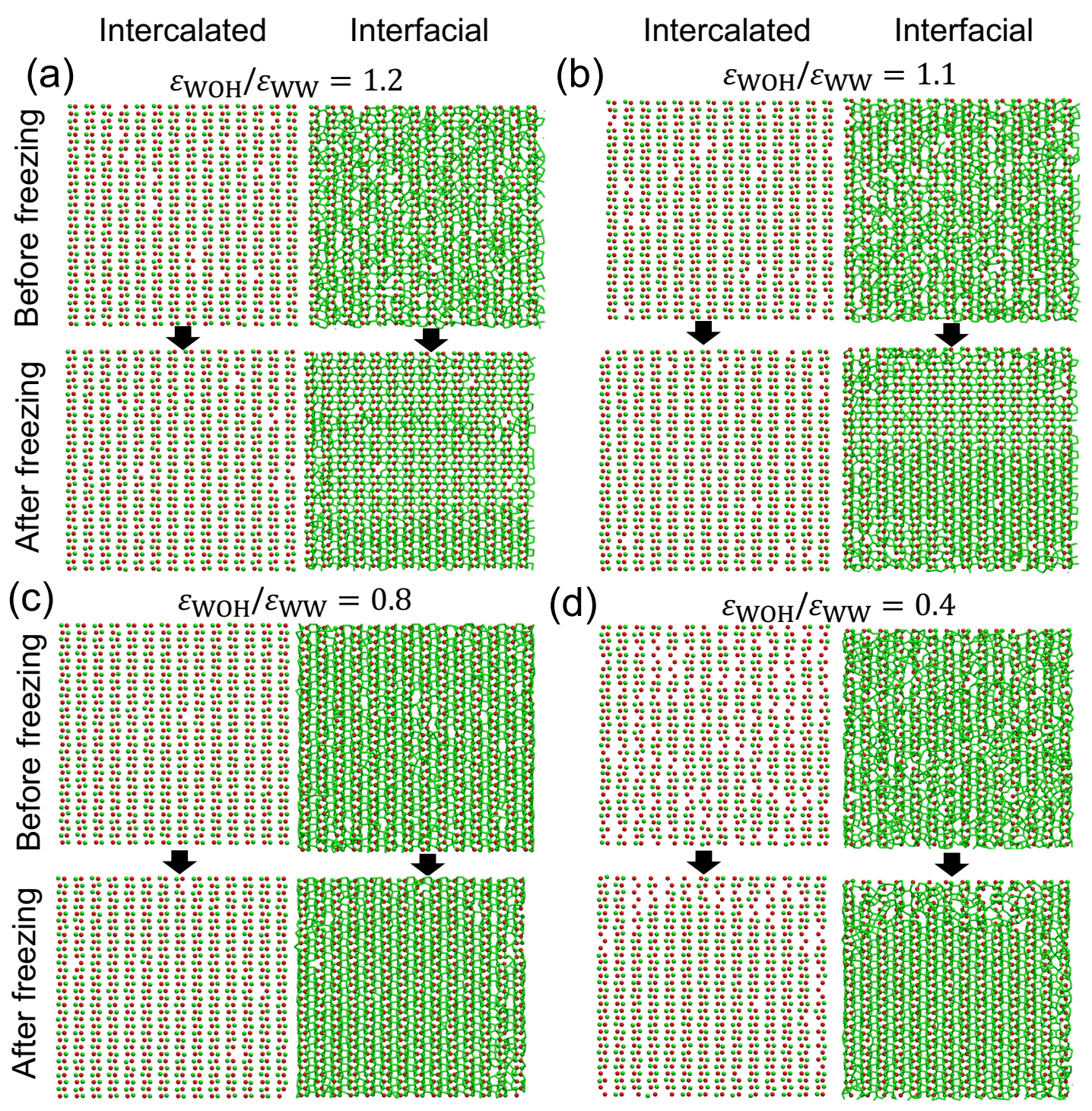

Fig. S5. Ordering of intercalated and interfacial water on the (010) faces of the phloroglucinol dihydrate crystal. Snapshots before and after ice crystallization for (a) $\varepsilon_{\mathrm{WOH}} / \varepsilon_{\mathrm{WW}}=1.2$, (b) $\varepsilon_{\mathrm{WOH}} / \varepsilon_{\mathrm{WW}}=1.1$, (c) $\varepsilon_{\mathrm{WOH}} / \varepsilon_{\mathrm{WW}}=0.8$, (d) $\varepsilon_{\mathrm{WOH}} / \varepsilon_{\mathrm{WW}}=0.4$. Hydroxyl groups of crystal and water molecules are denoted by red and green balls, respectively. We define the intercalated water as those within $0.1 \mathrm{~nm}$ and interfacial water as those within $0.35 \mathrm{~nm}$ form the plane of $\mathrm{OH}$ groups of organic crystal. 
$\Delta T_{f}=51 \pm 2 \mathrm{~K}$

$\delta a=-0.5 \%$ \& $\delta b=-0.4 \%$
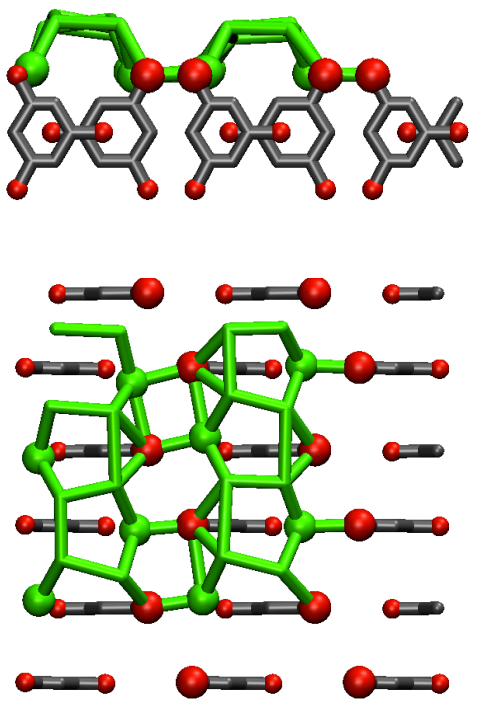

$\Delta T_{f}=33 \pm 2 \mathrm{~K}$

$\boldsymbol{\delta} a=-0.4 \% \& \delta b=5.8 \%$
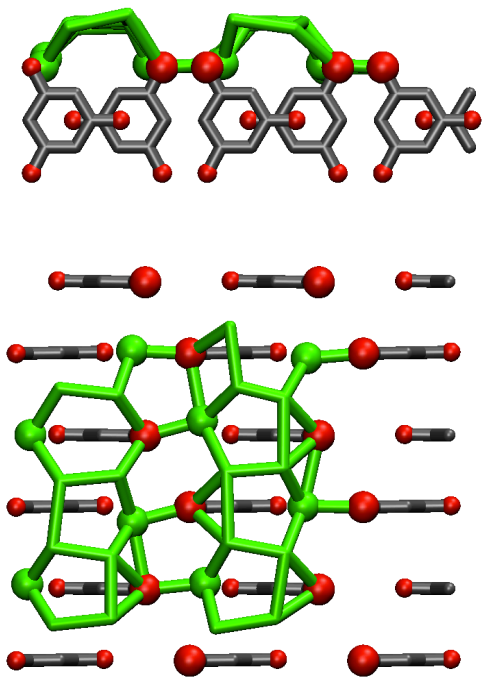

$\Delta T_{f}=47 \pm 2 \mathrm{~K}$

$\boldsymbol{\delta} a=-6.4 \% \& \delta b=-0.5 \%$

Fig. S6. Detail of the hydrogen-bonded rings of the ITL on the scaled phloroglucinol dihydrate crystals. OH groups are shown with red balls, intercalated water molecules with green balls. The aromatic carbons are shown in gray, and water and $\mathrm{OH}$ groups within $0.35 \mathrm{~nm}$ are connected by a green stick.

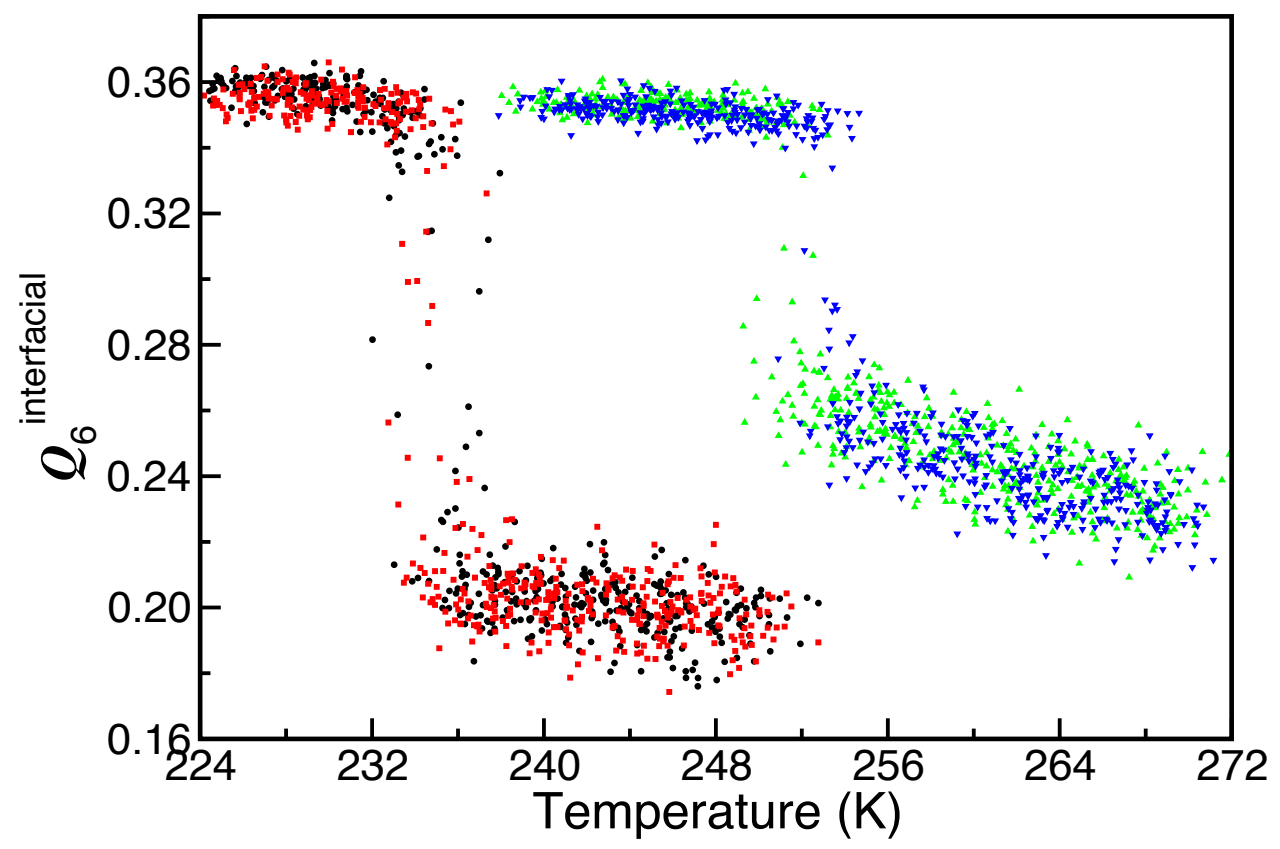

Fig. S7. Evolution of the ordering of interfacial water on the (010) face of scaled phloroglucinol dihydrate surfaces modeled with the united atom model and $\mathrm{mW}$ water in cooling simulations at a rate of $1 \mathrm{Kns}^{-1}$. Black and red points correspond to the surface with mismatch to ice $\delta a=-0.43 \%$ and $\delta b=5.75 \%$; green and blue points to the surface with mismatch $\delta a=-0.43 \%$ and $\delta b=-0.52 \%$. These two surfaces do not experience post-crystallization elimination of the ITL. 